\title{
Efficient generation of mouse ESCs-like pig induced pluripotent stem cells
}

Dear Editor,

Porcine induced pluripotency stem cells (piPSCs) are promised in basic research, animal husbandry and regenerative medicine. However, the efficiency of the piPSCs induction has been low and the generated piPSCs varied in cell morphology and cell characteristics. Here we report a novel approach to improve efficiency of piPSCs generation. The induced piPSCs are dome-shaped mouse embryonic stem cells (ESCs)-like and display molecular properties of mouse ESCs. Electroporation study reveals that mouse ESCs-like status facilitates genetic manipulating of piPSCs. Importantly, we demonstrate that the domed piPSC colonies are more suitable as donor cells for nuclear transfer (NT) to generate reconstructed embryos than those flattened piPSCs. The potential applications of the newly generated piPSCs in ungulate pluripotent research are discussed.

Previously, we generated the mouse ESCs-like human ESCs by modifying culture conditions (Gu et al., 2012). In this study, we generated the mouse ESCs-like piPSCs and enhanced induction rate of piPSCs by using LBX medium. The whole process of piPSCs generation was illustrated on Fig. 1A. The donor cells used for induction were pig embryonic fibroblasts (PEFs) that were obtained from 33.5 dpc embryos of Duroc strain. In LBX medium, the small colonies formed as early as the fifth day after induction, whereas, in KOSR medium, the colonies were firstly visualized at the eighth day after induction (Fig. 1B). We also tracked and compiled the number of colonies kinetically for five days and the efficiency of piPSCs formation in LBX medium was about five times of that in the KOSR medium (Fig. 1B). We then picked the colonies to derive some piPSCs lines. We compared piPSCs cultured in LBX medium with those cultured in KOSR medium and found some differences between them. Morphologically the former colony looked domed, just like mouse ESCs (these piPSCs were called pips_m cells), whereas, the later looked flattened (these piPSCs were called pips_h cells) (Fig. 1C). These cells also differed in cell viability and proliferation ability. The cells cultured in LBX medium had higher proliferation ability than those cultured in KOSR medium (Fig. 1D). Consistent with this observation, we also found that the capability of single cell colony formation was much stronger in LBX medium (Fig. 1E). The pips_m cells were predominantly diploid with normal 38 chromosome karyotypes (Fig. S1C) and expressed normal level of nuclear pluripotent marker OCT4 and membrane markers SSEA4, TRA-1-60 and SSEA1 (Fig. 1F), however, the pips_h cells did not express SSEA1 (Fig. S1A). We further detected the differentiation ability of these cells. Both types of the piPSCs could form embryonic bodies (EBs) in vitro when cultivated in suspension. The EBs also expressed markers of three germ layers (Fig. 1G) and had the neuron differentiation ability (Fig. 1H). The piPSCs could form teratomas when injected into immune-deficient mouse, and the teratomas were comprised of tissues from three germ layers (Fig. 1I). The detection of $X$ chromosome status for both type of piPSCs derived from the same female fibroblasts were carried out by H3K27 trimethyl staining, and we found that the most of pips_h cells had one inactive $X$ chromosome. As expected, both $X$ chromosomes were active in most of pips_m cells (Fig. S2).

Through Q-PCR analysis, we found that both the pips_m and pips_h cell lines expressed Oct4 and c-Myc transgenes (Oct4_v and $c-M y c \_v$ ), but the expression pattern of the transgenic Sox2 and Klf4 (Sox2_v and Klf4_v) were different between the pips_m and pips_h cell lines. The latter did not express Sox2_v but expressed Klf4_v continuously. The former had perpetual expression of Sox2_v and Klf4_v (Fig. $\mathrm{S} 3 \mathrm{~A})$. The expression of these four endogenous genes had also been detected. The expression of Oct4 and c-Myc (Oct4_3'UTR and c-Myc_3'UTR) were increased in all the lines of piPSCs, as compared with the original PEFs, but was lower than that of transgenic ones (Fig. S3A and S3B). As the methylation status of Oct4 promoter region is relevant to the gene expression of Oct4, the methylation status of promoter region of Oct4 were evaluated (Fig. S3C). Compared to the parthenogenetic blastocyst embryos (PA_Blastocyst), the methylation of Oct4 promoter in the pips_h and the pips_m cells was not similar to that in PA_Blastocyst. In addition to Oct4_3'UTR and c-Myc_3'UTR, endogenous Nanog and Sox2 (Nanog_3'UTR and Sox2_3'UTR) were measured as well. There was no difference in Sox2_3'UTR expression between pips_h and pips_m cells. Unexpected, the Nanog_3' UTR expression was higher in pips $\mathrm{h}$ cells than that in pips_m cells (Fig. S3B). Because the pips_h cells appeared similar to human ESCs colonies, whereas the pips_m cells looked like mouse ESCs colonies, we further defined the 
A

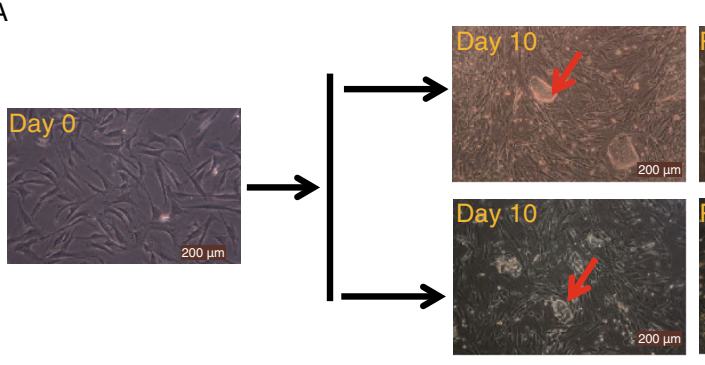

C
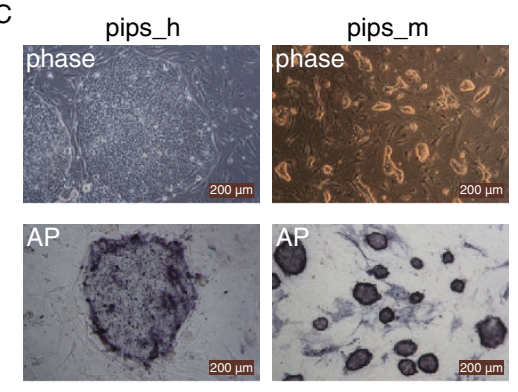

D
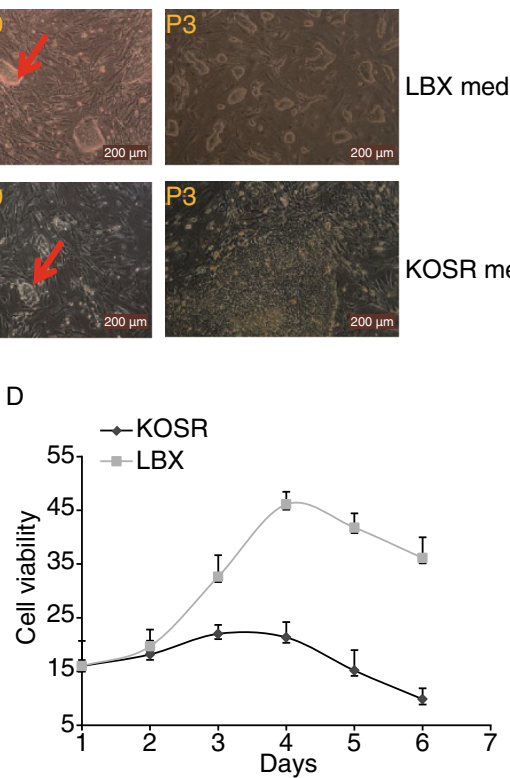

F

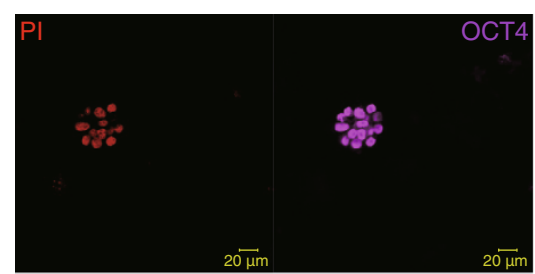

$20 \bar{m}$
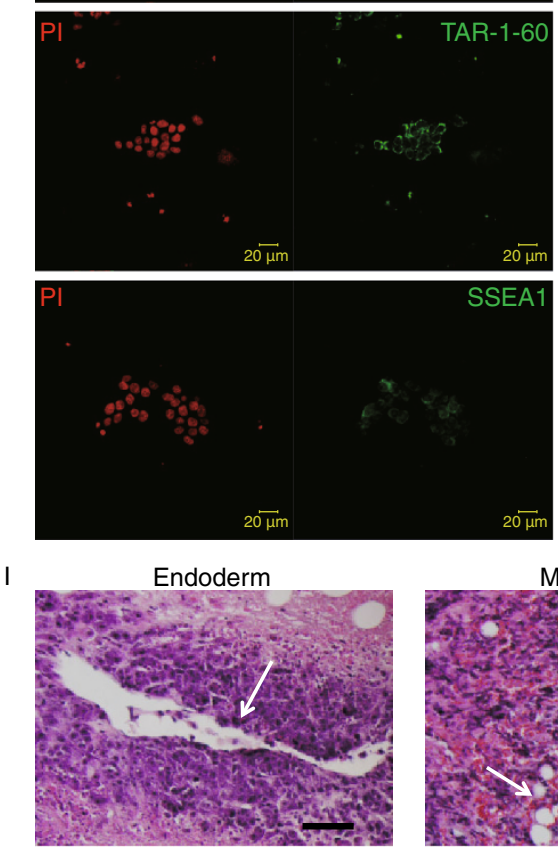

LBX medium

G
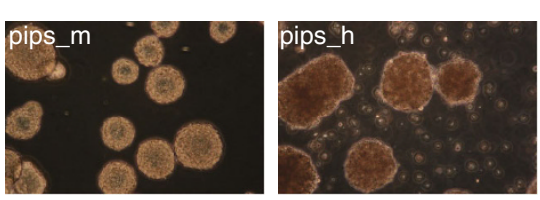

E
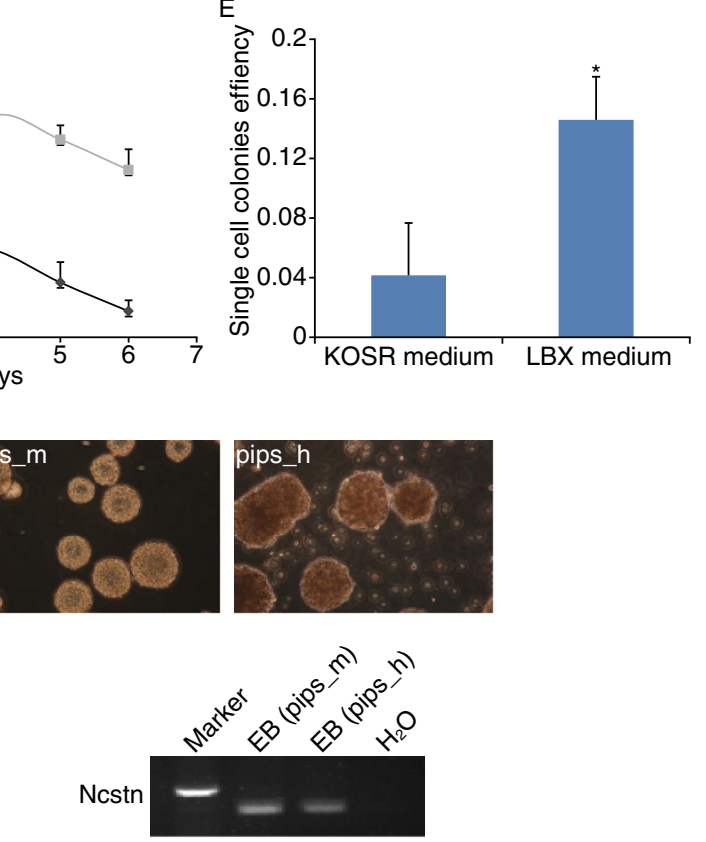

Osteonectin
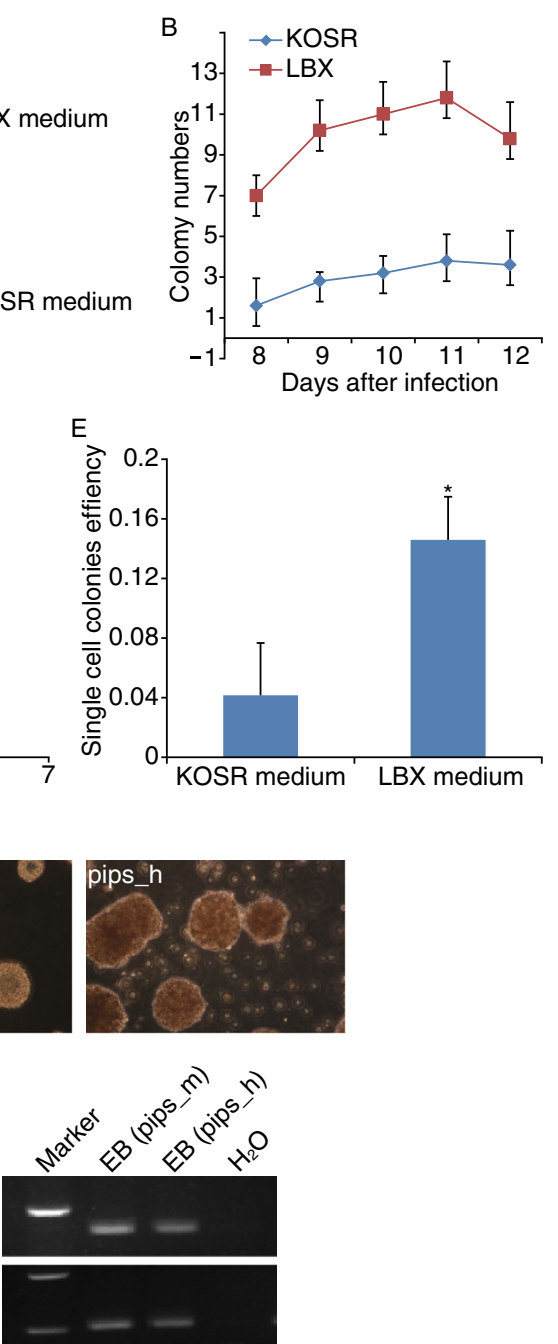

Neurod

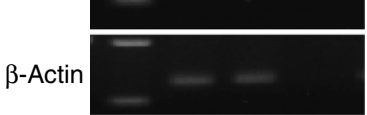

$\mathrm{H}$

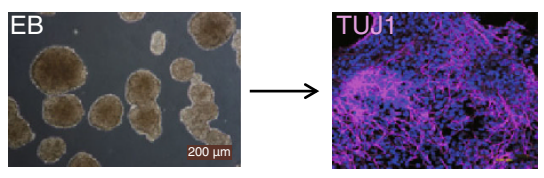


Figure 1. Efficient generation of mouse ESCs-like piPSCs in LBX medium. (A) Timeline for generating piPSCs in LBX and KOSR medium. Day 0 shows PEFs before infected with OSKM retrovirus, on the second day the infected PEFs were plated on MEF feeders with LBX and KOSR mediums. Day 10 shows PEFs infected 10 days later and colonies were yielded (red arrows). In LBX medium the colonies looked domed and were picked for digesting to derive cell lines. In KOSR medium the colonies looked flattened and were picked for cutting to derive cell lines. P3 shows the cell lines in KOSR and LBX mediums had been passaged for 3 times. Scale bars are $200 \mu \mathrm{m}$. (B) Colony number. Colonies were counted from 8 to 12 days after infection in LBX and KOSR medium, respectively. (C) Top, phase morphology of piPSCs derived in KOSR (pips_h) and LBX (pips_m) mediums. Bottom, piPSCs clones were stained with alkaline phosphatase kit. Scale bars are $200 \mu \mathrm{m}$. (D) Cell viability analysis for different cell lines. KOSR and LBX show the cell viability in KOSR and LBX medium. (E) Single cell colony formation. Cell lines derived in LBX medium had higher colony formation ability than that derived in KOSR medium. ${ }^{*} P<0.05$. (F) Pips_m cells clones were stained with pluripotency markers. Positive OCT4 (purple), SSEA4 (green), TRA-1-60 (green) and SSEA1 (green) were observed. DNA was stained with propidium iodide (PI, red). Shown were examples from pips-n-3. Scale bars are $20 \mu \mathrm{m}$. (G) In vitro embryoid body formation. Top, pips_m and pips_h cells (from pips-n-3 and pips-5 respectively) could form EB both, scale bars are $200 \mu \mathrm{m}$. Bottom, endoderm (Ncstn), mesoderm (Osteonectin) and ectoderm (Neurod) markers were detected by RT-PCR. $(H)$ Directed differentiation of pips_m cell derived EBs into neural linage. Left, EBs from examples of pips-n-3. Right, differentiated neurons were stained with Tuj1 (purple). DNA was stained with Hoechst (blue). (I) Teratoma formation of pips_m cells (from pips_m cell line pips-n-3). Tissues exhibiting all three germ layers were presented on the teratoma dissection slices identified by staining with haematoxylin and eosin. Left, endoderm with glands; Middle, mesoderm with fat tissues; Right, ectoderm with nervous tissues. Scale bars are $500 \mu \mathrm{m}$.

differences in signaling pathway between them. The expression of Lif, Lifr and Bmp4 were higher in the pips_m cells than that in the pips_h cells, Smad4 and Fgf2r expression were also higher in the pips_m cells than that in the pips_h cells (Fig. S3D). As with previously reported, the piPSCs expressed exogenous transgenic RNA. This was also the barrier of piPSCs development. There were no standard porcine ESCs generated up to now, so the research groups including us couldn't define what the piPSCs should be like. We tried hard to optimize the system to generate "better" piPSCs for application.

Stem cells are ideal donors for generation of transgenic animals by NT due to their ability of self-renewal and high resolution screening for positive genetically modified cells (Zhou et al., 2010). Up to now, no healthy cloned adults had been generated directly from piPSCs, but, differentiated piPSCs or small molecular compounds treated-piPSCs could support in vivo development of embryos and live-born (Fan et al., 2013b). We compared the development potency of pig NT embryos reconstructed from the pips_m and pips_h cells both in vitro and in vivo. The rate of in vitro blastocyst development was higher in the pips_m cell group compared to the pips_h cell group (Table S1). Some differences were also noted in vivo development. The surrogate mothers into whom the pips $h$ cell-derived embryos were introduced were not pregnant, whereas there were three pregnant receipts from four pips_m cell lines (Table S2). These findings are in agreement with recent reports that mouse ESCs-like state piPSCs could contribute to pig embryonic development (Fujishiro et al., 2013).

Importantly, the pips_m cells demonstrated good cell viability and could be digested into single cells and passaged easily. Therefore they were suitable for transgenic application. We electroporated the pips_m and pips_h cells equally with the modified PiggyBac vector carrying red fluorescence protein (RFP) and neomycin-resistant (neo ${ }^{r}$ ) genes (Fig. S4A). Four days after the neomycin-resistant colonies appeared. Our results showed that the number of colonies yielded from the pips_m cells were 40 folds higher than that yielded from the pips_h cells with the same amount of the vector (Table 1). In addition, more colonies for the pips_h cells were noted after electroporation treated with Y27632 (Rock inhibitor). We randomly picked 30 pips_m neomycinresistant colonies and 26 of them had been expanded to become cell lines (Fig. S4B). We also picked equal number of pips_h neomycin resistant colonies, however, only four colonies formed cell lines (Fig. S4C). Therefore the pips_m might have higher potential in transgene application.

High efficiency and availability of pluripotent stem cells are essential for their application in domestic ungulates. Some new genes had been involved to enhance piPSCs induction (Fan et al., 2013a; Petkov et al., 2013). In this work, we produced the pips_m cells with high efficiency by optimizing the culture medium. We concluded that the small molecular compounds in LBX medium contributed to the formation of mouse ESCs like colonies and improved cell viability. It was reported that these small molecular compounds had been used for converting human ESCs into mouse ESCs-like state and can maintain long-term survival of human ESCs (Hanna et al., 2010; Gu et al., 2012). After removing the small compounds from LBX medium, the colonies had a tendency to become flattened (Wang et al., 2013), suggesting that these small molecular compounds are responsible for mouse ESCs-like colony formation and cell proliferation. Another report also showed that a small molecular compound, Forskolin, could contribute to the generation of the naïve state of piPSCs (Fujishiro et al., 2013). During our work progress, other independent studies also found LIF and some inhibitors could modulate the pluripotency of piPSCs (Cheng et al., 2012; Cloke et al., 2012; Kwon et al., 2013). Nonetheless, this was a novel way to derive piPSCs and also had a comprehensive comparison of 
Table 1. Electroporation of transgenes into pips_m and pips_h cells

\begin{tabular}{lllll}
\hline Cell lines & & $\begin{array}{l}\text { Cells for } \\
\text { electroporation }\end{array}$ & Electroporation & $\begin{array}{l}\text { Average colonies } \\
\text { after drug selection }\end{array}$ \\
\hline pips_m & pips_n_3 & $1.1 \times 10^{6}$ & 3 & $>4400$ \\
& pips_n_5 & $1.2 \times 10^{6}$ & 3 & $>4580^{*}$ \\
& pips_n_6 & $1.0 \times 10^{5}$ & 2 & $>580$ \\
pips_h & pips_n_7 & $6.4 \times 10^{5}$ & 3 & 120 \\
& pips_1 & $1.1 \times 10^{6}$ & 3 & $500^{*}$ \\
& pips_2 & $1.2 \times 10^{6}$ & 3 & 40 \\
& pips_5 & $1.0 \times 10^{5}$ & 3 & 3 \\
\end{tabular}

* Cells were treated with Y27632 after electroporation.

piPSCs for the two states. In our study, the pips_m cells had higher transgenic efficiency which indicated that the mouse ESCs-like state facilitated the transgenic manipulation (Buecker et al., 2010). As human ESCs morphologically were more similar to mouse EpiSCs, many studies had paid attention to the pluripotency of human ESCs and to converting human ESCs to mouse ESCs-like (Gu et al., 2012). Because of the ethical limitation, it was not possible to perform embryonic experiments to determine the pluripotency of human ESCs and to find that the converted human ESCs were prone to mouse ESCs at both phenotype and internality levels (Li and Ding, 2011; Young, 2011). Our results involved pluripotent research in other species. Domed colonies of piPSCs might have better developmental potency in vitro and in vivo than the flattened ones.

In summary, we were able to efficiently produce mouse ESCs-like piPSCs. The piPSCs had good cell viability and proliferation capability. Exogenic pancreas had been successfully generated using blastocyst complementation in pigs (Matsunari et al., 2013). Our study will provide a novel tool for further transgenic application and naïve states exploration of pig pluripotent cells.

\section{FOOTNOTES}

This study was supported in part by grants from the National Basic Research Program (973 Program) (Nos. 2011CB944102 and 2012CBA01300), and Ministry of Agriculture China (Major transgenic project) 2011ZX08010-001. We would like to thank Zhilian Yue (IPRI, UOW) for her native grammar correction.

Qi Gu, Jie Hao, Tang Hai, Jianyu Wang, Yundan Jia, Qingran Kong, Juan Wang, Chunjing Feng, Binghua Xue, Bingteng Xie,

Electronic supplementary material The online version of this article (doi:10.1007/s13238-014-0043-2) contains supplementary material, which is available to authorized users.

Qi Gu, Jie Hao, Tang Hai, and Jianyu Wang contributed equally to this work.
Shichao Liu, Jinyu Li, Yilong He, Jialu Sun, Lei Liu, Liu Wang, Zhonghua Liu and Qi Zhou declare that they have no conflict of interest. All institutional and national guidelines for the care and use of laboratory animals were followed.

Qi Gu ${ }^{1,3}$, Jie Hao ${ }^{1}$, Tang Hai ${ }^{1}$, Jianyu Wang ${ }^{1,2}$, Yundan Jia ${ }^{1}$, Qingran Kong ${ }^{2}$, Juan Wang ${ }^{1,2}$, Chunjing Feng ${ }^{1}$, Binghua $\mathrm{Xue}^{2}$, Bingteng $\mathrm{Xie}^{2}$, Shichao $\mathrm{Liu}^{2}$, Jinyu $\mathrm{Li}^{2}$, Yilong $\mathrm{He}^{2}$, Jialu Sun ${ }^{2}$, Lei Liu ${ }^{1}$, Liu Wang ${ }^{1}$, Zhonghua Liu ${ }^{2 凶}$, Qi Zhou ${ }^{1 凶}$

${ }^{1}$ State Key Laboratory of Reproductive Biology, Institute of Zoology, Chinese Academy of Sciences, Beijing 100101, China

${ }^{2}$ College of Life Science, Northeast Agricultural University of China, Harbin 150030, China

${ }^{3}$ Intelligent Polymer Research Institute, ARC Center of Excellence for Electromaterials Science, AlIM Facility, University of Wollongong, Wollongong, NSW 2522, Australia

$\bowtie$ Correspondence: liu086@126.com (Z. Liu), zhouqi@ioz.ac.cn (Q. Zhou)

\section{OPEN ACCESS}

This article is distributed under the terms of the Creative Commons Attribution License which permits any use, distribution, and reproduction in any medium, provided the original author(s) and the source are credited.

\section{REFERENCES}

Buecker C, Chen HH, Polo JM, Daheron L, Bu L, Barakat TS, Okwieka P, Porter A, Gribnau J, Hochedlinger K et al (2010) A murine ESClike state facilitates transgenesis and homologous recombination in human pluripotent stem cells. Cell Stem Cell 6:535-546

Cheng D, Guo Y, Li Z, Liu Y, Gao X, Gao Y, Cheng X, Hu J, Wang H (2012) Porcine induced pluripotent stem cells require LIF and maintain their developmental potential in early stage of embryos. PLoS ONE 7:e51778

Cloke T, Munder M, Taylor G, Müller I, Kropf P (2012) Modulation of pluripotency in the porcine embryo and iPS cells. PLoS ONE 7: e49079 
Fan A, Ma K, An X, Ding Y, An P, Song G, Tang L, Zhang S, Zhang P, Tan $W$ et al (2013a) Effects of TET1 knockdown on gene expression and DNA methylation in porcine induced pluripotent stem cells. Reproduction 146:569-579

Fan N, Chen J, Shang Z, Dou H, Ji G, Zou Q, Wu L, He L, Wang F, Liu $\mathrm{K}$ et al (2013b) Piglets cloned from induced pluripotent stem cells. Cell Res 23:162-166

Fujishiro SH, Nakano K, Mizukami Y, Azami T, Arai Y, Matsunari H, Ishino R, Nishimura T, Watanabe M, Abe T et al (2013) Generation of naive-like porcine-induced pluripotent stem cells capable of contributing to embryonic and fetal development. Stem Cells Dev 22:473-482

Gu Q, Hao J, Zhao XY, Li W, Liu L, Wang L, Liu ZH, Zhou Q (2012) Rapid conversion of human ESCs into mouse ESC-like pluripotent state by optimizing culture conditions. Protein Cell 3:71-79

Hanna J, Cheng AW, Saha K, Kim J, Lengner CJ, Soldner F, Cassady JP, Muffat J, Carey BW, Jaenisch R (2010) Human embryonic stem cells with biological and epigenetic characteristics similar to those of mouse ESCs. Proc Natl Acad Sci USA 107:9222-9227

Kwon DJ, Jeon H, Oh KB, Ock SA, Im GS, Lee SS, Im SK, Lee JW, Oh SJ, Park JK et al (2013) Generation of leukemia inhibitory factor-dependent induced pluripotent stem cells from the Massachusetts General Hospital miniature pig. BioMed Res Int 2013:140639

Li W, Ding S (2011) Human pluripotent stem cells: decoding the naive state. Sci Transl Med 3:76ps10

Matsunari H, Nagashima H, Watanabe M, Umeyama K, Nakano K, Nagaya M, Kobayashi T, Yamaguchi T, Sumazaki R, Herzenberg LA et al (2013) Blastocyst complementation generates exogenic pancreas in vivo in apancreatic cloned pigs. Proc Natl Acad Sci USA 110:4557-4562

Petkov S, Hyttel P, Niemann H (2013) The choice of expression vector promoter is an important factor in the reprogramming of porcine fibroblasts into induced pluripotent cells. Cell Reprogram 15:1-8

Wang J, Gu Q, Hao J, Jia Y, Xue B, Jin H, Ma J, Wei R, Hai T, Kong $Q$ et al (2013) Tbx3 and Nr5alpha2 play important roles in pig pluripotent stem cells. Stem Cell Rev 9:700-708

Young RA (2011) Control of the embryonic stem cell state. Cell 144:940-954

Zhou S, Ding C, Zhao X, Wang E, Dai X, Liu L, Li W, Liu Z, Wan H, Feng $C$ et al (2010) Successful generation of cloned mice using nuclear transfer from induced pluripotent stem cells. Cell Res 20:850-853 